\title{
BILINGUAL VARIABLE MESSAGE SIGNS: A STUDY OF INFORMATION PRESENTATION AND DRIVER DISTRACTION
}

\author{
Samantha L. Jamson \\ Fergus N. Tate \\ A. Hamish Jamson \\ Institute for Transport Studies \\ University of Leeds \\ Leeds, U.K. \\ E-mail:sjamson@its.leeds.ac.uk
}

\begin{abstract}
Summary: Research on static bilingual signs has confirmed increased reading times compared to their monolingual counterparts; however there is little empirical research on bilingual Variable Message Signs (VMS). The study reported here evaluates the effect of various bilingual VMS configurations on driver behaviour and safety. The aim of the study was to determine whether or not the visual distraction associated with bilingual VMS signs of different configurations (length, complexity) impacted on driving performance. The results suggest that four-line bilingual VMS signs comprising 2 lines of text in each language are read by both monolingual and bilingual drivers in a manner that more closely approximates a two-line monolingual sign. This being the case it is likely that the deployment of four-line bilingual signs on Welsh motorways is unlikely to result in a significant reduction in safety.
\end{abstract}

\section{INTRODUCTION}

The Welsh language is the oldest living language of Great Britain and one of the oldest in Europe. At the start of the 20th century, the Welsh language was spoken by almost half the

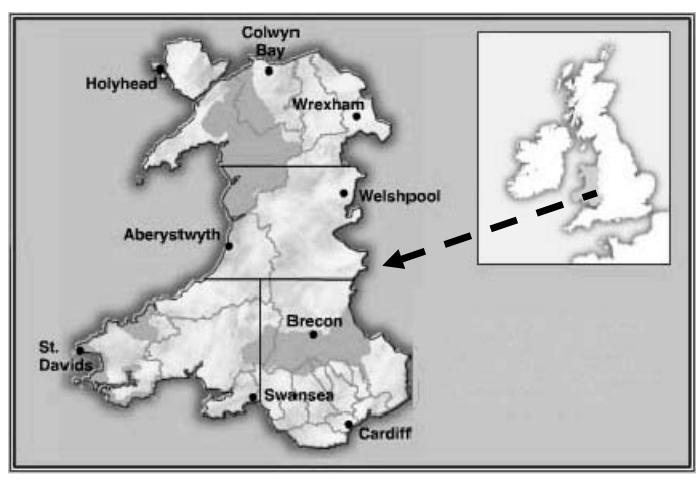
population of Wales. However, since then the number of Welsh speakers has decreased steadily until fairly recently. By 1991, although the number of people able to speak the language was still more than half a million, this represented just $18.7 \%$ of the population. The 1991 Census results suggested that the decline has stabilised, particularly among young people. It is likely that the 2001 Census will show that there has been a further increase, because nearly one-third of all primary school children in Wales are now receiving their education in Welsh-medium or bilingual schools. As a result of the Government of Wales Act 1998, both Welsh and English are official languages of the National Assembly for Wales. The Assembly is required to treat both languages equally, so far as is practicable, in conducting all its business, and all legislation must be in both languages. Traffic signs are part of this legislation.

Research on static bilingual signs, using the same character sets, has confirmed increased reading times compared to their monolingual counterparts (e.g. Harjula, Luoma and Rämä, 1998). This is a simple reflection of the fact that reading time increases with the number of words displayed on the sign. Additional research (Rutley, 1972; Rutley, 1974) found that overall reading time was minimised when English was placed uppermost on the sign. 
Current practice in Wales is to use the dominant language on the first line of the sign and the secondary language on the second line. Thus Welsh text typically appears at the top of signs in North Wales where the Welsh language is more widely spoken, while English appears first in South Wales.

Although bilingual static traffic signs have been used in Wales for more than a decade, the move to bilingual Variable Message Signs (VMS) is new. VMS can convey a wide range of messages, which may be varied temporally in response to the prevailing traffic and environmental conditions. VMS are therefore an extremely powerful traffic management tool. Research at a European level has attempted to produce guidelines for best practice (e.g. TROPIC), however basic research has generally concentrated on only one implementation of a sign. As such, little comparative work has been carried out.

When presenting bilingual information to drivers using VMS signs, there are three possible options.

Bilingual signs whereby the complete English and Welsh text appears on the same sign. However, monolingual drivers have to search the sign in order to locate the appropriate information.

FOR MIDLANDS USE (M4) M5

I MIDLANDS DILYN (M4) M5

$>$ Sequenced monolingual signs where one sign presents the message in one language and another sign presents the same message in the other language. However, monolingual drivers are faced with a message that is not recognisable. Furthermore, because the character forms are the same for English and Welsh, the drivers' initial reaction will be an attempt to "read" the message before identifying that it is not recognisable.

$>$ Split bilingual signs where a message is split over consecutive bilingual signs. These could, in theory, overcome the limitations posed by sequenced monolingual signs. While overcoming the problems identified with one group being provided with information before another, such signs could increase confusion or cognitive load as drivers seek to decide what response is required, when given only part of a message.

In the study presented here, the following research questions will be studied.

- Do drivers slow down to read the signs, and if so, which ones?

- Is drivers' ability to maintain "safe" headway affected by reading the signs?

- Are there any differences in behaviour with monolingual and bilingual signs?

- Do four-line bilingual signs have an additional adverse effect?

The key outcome of the simulator experiments will be to determine whether or not the visual distraction associated with bilingual VMS signs of different configurations (length, complexity) impacts on driving performance. 


\section{METHOD}

\section{Experimental Design}

Participants in the study were required to drive the experimental route twice. On each of these occasions, drivers were asked to follow a lead car until they reached the end of the route. They were asked not to overtake this car and to remain at all times in the left hand lane. The difference between the two routes lay in the behaviour of the lead car. On one of the routes this car remained at a constant speed (approximately $70 \mathrm{mph}$ ). On the other route, the lead car varied its speed in the vicinity of the signs. This was considered as a higher workload environment than the previous route as drivers were not only required to read the signs but also adapt their speed to maintain a safe headway to the car in front. Two types of messages were included, traffic-related messages and instructional messages to which the driver had to respond using the vehicle controls and thereby registering a response. In order to minimise potential learning effects some changes between the traffic runs were made.

\section{Participants}

Twenty four participants took part in the experiment. This sample comprised of an equal number of monolinguals (English) and bilinguals (English and Welsh). There were no monolingual Welsh participants in the sample.

\section{Road Environment}

A two-lane road of motorway design standard was used for the experimental route. It was comprised of straight sections of road 500 metres long separated by "filler" pieces of road. The signs were located on the 500 metres stretches of road with the sign triggered to activate 50 metres from start and the sign located 300 metres from start. The road was approximately $30 \mathrm{~km}$ in length, with a light volume of surrounding traffic. In the outside lane occasional vehicles travelling at $80 \mathrm{mph}$ passed the driver. Snapshots of the scene are shown in Figures 1

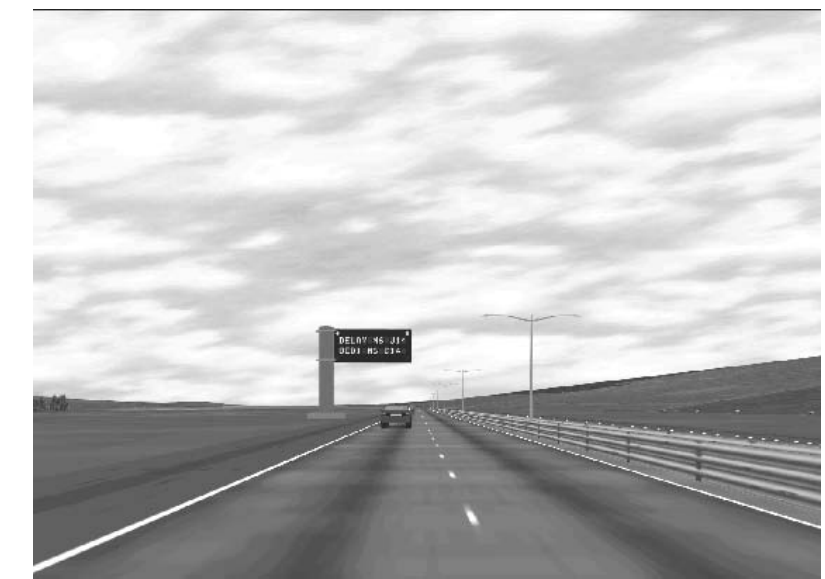

and 2.

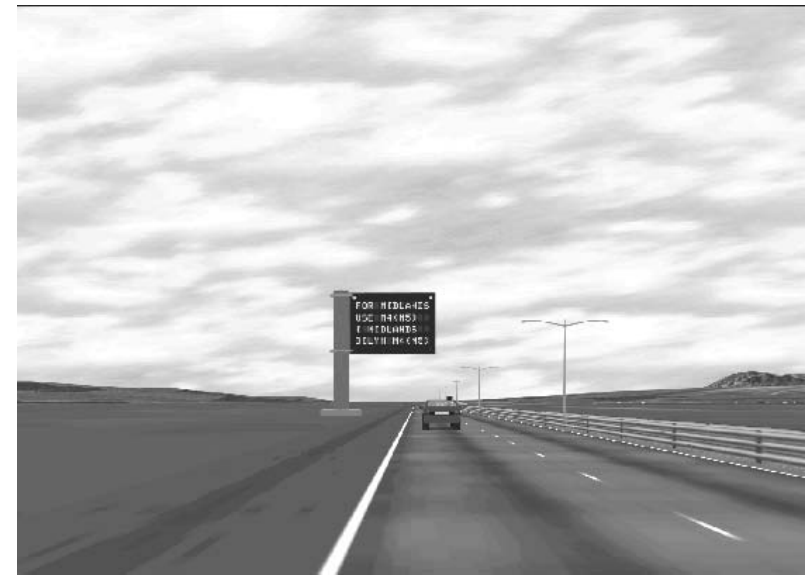

Figure 2: Four-line VMS

\section{Data Collection}

Driver performance was measured using data on speed and speed variation, lateral position and headway to a lead vehicle. The data (speed and headway) were collected on the four 
sections (Figure 3). Measures of mental workload were collected using the NASA-RTLX questionnaire. This measures subjective mental workload on six separate dimensions.

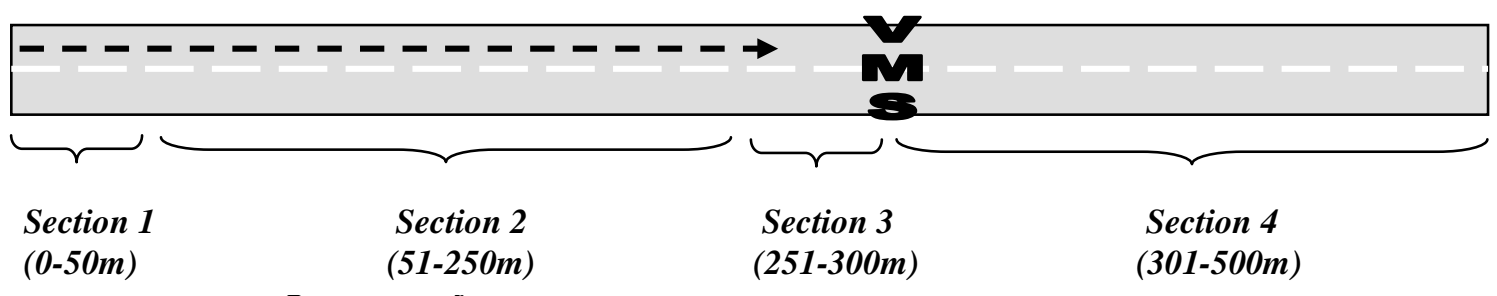

Figure 3: Data collection

\section{RESULTS}

\section{Workload}

It was found that there were significant differences reported between the workload conditions. Workload scores for mental demand $(\mathrm{F}[1,47]=11.74, \mathrm{p}<.001)$, performance $(F[1,47]=12.56, \mathrm{p}<.001)$ and effort $(\mathrm{F}[1,47]=11.79, \mathrm{p}<.001)$ were all higher in the high workload condition.

\section{Mean Speed}

Mean speeds were calculated across each of the four sections in the vicinity of each of the signs. The number of lines of text and subject type (monolingual or bilingual), were included as factors. No differences were found in mean speeds according to subject type. Overall there was a main effect of Section on speed $(F[3,2157]=20.34, \mathrm{p}<.001)$, i.e. speed changes were observed through the 500m stretches of road (independent of sign type). Post hoc comparisons (Tukey) showed that mean speeds in Section 2 (i.e. the period for which the driver was engaged in reading the signs) were lower than in all other sections. Mean speeds in Sections 1 and 3 were comparable; thus drivers returned to initial speed after reading the sign. 
Number of lines of text. Whilst the above analysis is interesting as an overview of the data, the study aimed to differentiate driver performance in terms of message type. Differences in mean speed (across all sections) were found according to the number lines of text present on the signs. $(\mathrm{F}[3,716]=4.61, \mathrm{p}<.01)$. Post hoc analyses showed that reductions in mean speed in

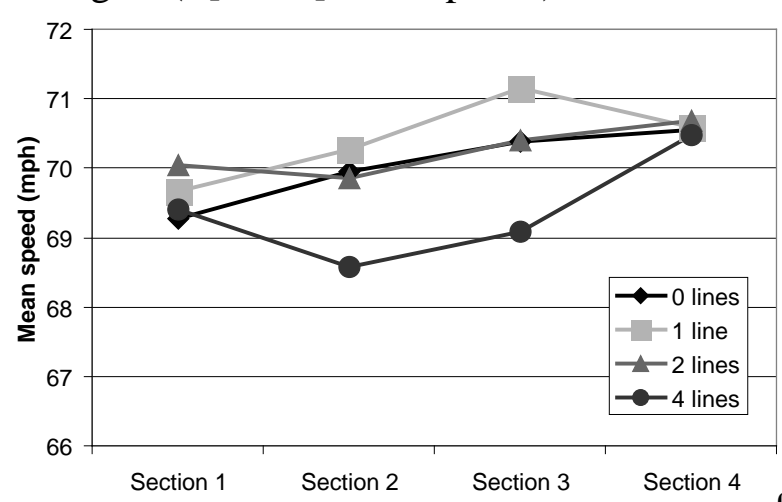

hangs over into Section 3. the vicinity of signs were only present for signs with 4 lines of text displayed (Figure 4). From the first analysis performed it appears that most of the effects of the signs are observed in Section 2 (logical considering this is where the sign is activated). Indeed Figure 4 illustrates that for signs with 2 or fewer lines of text displayed, mean speeds are comparable in Sections 1 and 4. Only in Section 2 does the difference for the 4 line signs become apparent; this effect

\section{Figure 4: Interaction between location and sign complexity}

Number of languages. By splitting four-line signs by number of languages (1 or 2), an analysis can be undertaken to discover whether a four-line sign comprising 2 languages is any different in terms of driver performance from a four-line sign of 1 language. When mean

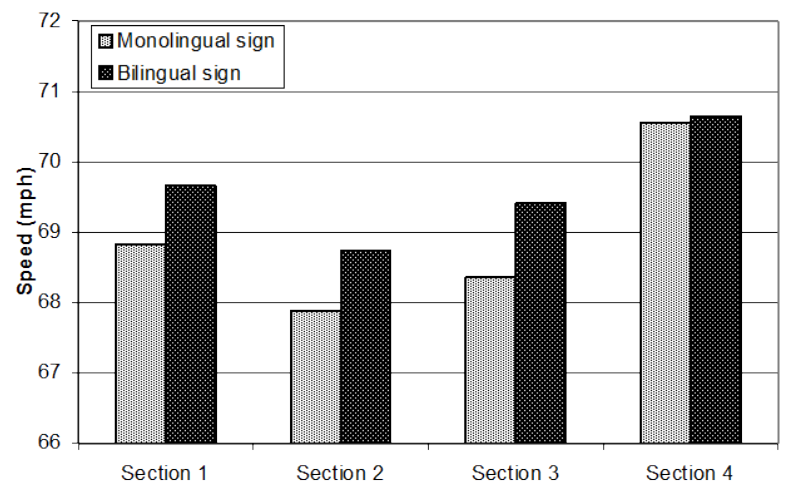

least attempting to do so, see Figure 5. speed was analysed, it was found that there were no significant differences between the behaviour of drivers in response to these two types of sign, even though half of the information displayed in the bilingual sign was not relevant to the drivers. Furthermore there were no differences between monolingual and bilingual drivers. This suggests that all the drivers were either reading the Welsh component of the sign, or in the case of the monolinguals, at the very

\section{Figure 5: Mean speed for four-line signs (split by number of languages)}

Arrangement of languages. On four-line bilingual signs there are a number of arrangements of the text that could be implemented. The text can be arranged by language or by message content. An analysis was undertaken to discover the effects each of these configurations has on driver behaviour. Only four-line bilingual signs were included in the analysis. No differences were found between the measures, such that mean speeds, standard deviation speed, and minimum headway (by section) were the same. This was true for both the high and low workload conditions.

\section{Car Following}

The car following test measures the ability of drivers to maintain a safe headway to a vehicle in front (Brookhuis et al 1987). Only headways of less than 6 seconds were used. Higher values assume that drivers are not intending to follow the lead vehicle. No statistically significant differences were found in either of the measures when the number of lines of text 
on the signs was taken into account. This implies that drivers' ability to maintain and adjust their headway was not disrupted by the requirement to read the signs.

\section{CONCLUSIONS}

In the overall analysis there were no differences in driver performance between the monolinguals and the bilinguals. Both were able to read the two-line signs with no compensatory effects in their speed. However, four-line bilingual signs did impact on driving performance in that mean speed across the section of the road where the sign was visible was significantly decreased. This is likely to be due to drivers adapting their speed in order in increase the available reading time.

The simulator study confirmed that drivers seeking to read a four-line monolingual sign reduce speed significantly more than those reading a two-line monolingual sign. Neither the speed or following headways recorded while reading a four-line bilingual sign were significantly different to those recorded for both the two-line monolingual or those recorded when reading a four-line monolingual sign. The implication is that the four-line bilingual sign lies "in the middle" of these two (in terms of reading difficulty). However the speed changes were typically less than $1.5 \mathrm{mph}$, which at a mean speed of $70 \mathrm{mph}$ and over a distance of 250 metres equates to a deceleration of $0.08 \mathrm{~ms}^{2}$. While statistically significant, in practical terms is unlikely to cause disruptions in traffic flow, except at high traffic densities when the mean speed will be much less and so will the deceleration.

In conclusion it appears that four-line bilingual VMS signs comprising 2 lines of text in each language are read by both monolingual and bilingual drivers in a manner that more closely approximates a two-line monolingual sign. This being the case it is likely that the deployment of four-line bilingual signs on Welsh motorways is unlikely to result in a significant reduction in safety.

\section{REFERENCES}

Brookhuis, K.A., Volkerts, E.R. and O’Hanlon, J.F. (1987). The effects of some anxiolytics on car following performance in real traffic. In Alcohol, Drugs and Traffic Safety - T86, Noordzij PC, Roszbach R (eds). Excerpta Medica: Amsterdam; 223-226.

Harjula, V., Luoma, J., and Rämä, P. (1998). Effects of Bilingual Variable Message Signs on Information Overload. TROPIC project. EC Contract RO-96-SC.303/2.

Rutley, K. (1972). An investigation into bilingual (Welsh/English) Traffic signs. TRRL Report LR 475. Transport Research Laboratory, Crowthorne, UK.

Rutley, K. (1974). An second investigation into bilingual (Welsh/English) Traffic signs. TRRL Supplementary Report 34 UC. Transport Research Laboratory, Crowthorne, UK. 